MATHEMATICS OF COMPUTATION

Volume 69, Number 232, Pages 1521-1532

S 0025-5718(99)01170-9

Article electronically published on August 24, 1999

\title{
BIVARIATE COMPOSITE VECTOR VALUED RATIONAL INTERPOLATION
}

\author{
JIEQING TAN AND SHUO TANG
}

\begin{abstract}
In this paper we point out that bivariate vector valued rational interpolants (BVRI) have much to do with the vector-grid to be interpolated. When a vector-grid is well-defined, one can directly design an algorithm to compute the BVRI. However, the algorithm no longer works if a vector-grid is ill-defined. Taking the policy of "divide and conquer", we define a kind of bivariate composite vector valued rational interpolant and establish the corresponding algorithm. A numerical example shows our algorithm still works even if a vector-grid is ill-defined.
\end{abstract}

\section{Motivation}

Let $\left\{\left(x_{i}, y_{j}\right) \mid i, j=0,1, \ldots, n\right\}$ be a set of points in $\mathbb{R}^{2}$ and let these points be reordered into the following array

$$
\begin{array}{ccc}
\left(x_{0}, y_{0}\right) & \cdots & \left(x_{0}, y_{n}\right) \\
\vdots & \ddots & \vdots \\
\left(x_{n}, y_{0}\right) & \cdots & \left(x_{n}, y_{n}\right)
\end{array}
$$

where $x_{i}>x_{i+1}$ and $y_{i}<y_{i+1}$ for $i=0,1, \cdots, n-1$. We call this array a square point-grid, and denote it by $\Pi^{n}$. Let $\vec{v}_{i, j} \in \mathbb{C}^{d}$ be a $d$-dimensional vector associated with the point $\left(x_{i}, y_{j}\right) \in \Pi^{n}$. Similarly we arrange these $\vec{v}_{i, j}$ into the following array

$$
\begin{array}{cccc}
\vec{v}_{0,0} & \vec{v}_{0,1} & \cdots & \vec{v}_{0, n} \\
\vec{v}_{1,0} & \vec{v}_{1,1} & \cdots & \vec{v}_{1, n} \\
\vdots & \vdots & \ddots & \vdots \\
\vec{v}_{n, 0} & \vec{v}_{n, 1} & \cdots & \vec{v}_{n, n}
\end{array}
$$

and call it a vector-grid, denoted by $\vec{V}^{n}$.

For a $d$-dimensional vector $\vec{v}=\left(v_{1}, v_{2}, \cdots, v_{d}\right) \in \mathbb{C}^{d}$, its generalized inverse (or the Samelson inverse) is defined as (see [3]

$$
\vec{v}^{-1}=\frac{\left(v_{1}^{*}, v_{2}^{*}, \cdots, v_{d}^{*}\right)}{\sum_{i=1}^{d} v_{i} v_{i}^{*}},
$$

where $v_{i}^{*}$ denotes the complex conjugate of $v_{i}$.

Received by the editor April 10, 1997 and, in revised form, December 10, 1998.

1991 Mathematics Subject Classification. Primary 41A20; Secondary 65D05.

Key words and phrases. Branched continued fraction, interpolation, algorithm.

Supported by the National Natural Science Foundation of China and in part by the Spanningthe-Century Foundation for Excellent Talents of the Ministry of the Machine Building Industry of China.

(C)2000 American Mathematical Society 
Definition 1.1. A $d$-dimensional vector valued polynomial

$$
\vec{N}(x, y)=\left(N_{1}(x, y), N_{2}(x, y), \cdots, N_{d}(x, y)\right)
$$

is said to be of degree $n$ and we write $\partial \vec{N}=n$, if $\partial N_{i}(x, y) \leq n$ for $i=1,2, \cdots, d$ and $\partial N_{j}(x, y)=n$ for some $j(1 \leq j \leq d)$.

Definition 1.2. Denote by $H_{n}$ the collection of all bivariate polynomials with total degree not exceeding $n$ and by $\vec{H}_{n}$ the collection of $d$ dimensional bivariate vector valued polynomials of degree $n$. Then the set

$$
\vec{H}_{n, m}=\left\{\vec{N}(x, y) / M(x, y) \mid \vec{N}(x, y) \in \vec{H}_{n}, M(x, y) \in H_{m}\right\}
$$

is called the collection of bivariate vector valued rational functions of type $(n / m)$.

All vectors in this paper are $d$-dimensional unless otherwise specified.

Making use of the Samelson inverse and reciprocal difference, one of the authors constructed the following Thiele-type branched continued fraction (see [5]):

$$
\vec{r}_{n}(x, y)=\vec{t}_{0}(y)+\frac{x-x_{0}}{\vec{t}_{1}(y)}+\cdots+\frac{x-x_{n-1}}{\vec{t}_{n}(y)}
$$

where

$$
\begin{aligned}
\vec{t}_{l}(y) & =\vec{c}_{l, 0}\left(\overline{x_{0}, \cdots, x_{l}} ; y_{0}\right) \\
& +\frac{y-y_{0}}{\vec{c}_{l, 1}\left(\overline{x_{0}, \cdots, x_{l}} ; y_{0}, y_{1}\right)}+\cdots+\frac{y-y_{n-1}}{\vec{c}_{l, n}\left(\overline{x_{0}, \cdots, x_{l}} ; y_{0}, \cdots, y_{n}\right)},
\end{aligned}
$$

and $\vec{c}_{i, j}\left(\overline{x_{0}, \cdots, x_{i}} ; y_{0}, \cdots, y_{j}\right)$ are computed through the following recursive process

$$
\vec{c}_{0,0}\left(\bar{x}_{i}, y_{j}\right)=\vec{v}_{i, j} \quad(i=0,1, \cdots, n, j=0,1, \cdots, n),
$$

$$
\vec{c}_{0, j}\left(\bar{x}_{i} ; y_{0}, \cdots, y_{j}\right)=\frac{y_{j}-y_{j-1}}{\vec{c}_{0, j-1}\left(\bar{x}_{i} ; y_{0}, \cdots, y_{j-2}, y_{j}\right)-\vec{c}_{0, j-1}\left(\bar{x}_{i} ; y_{0}, \cdots, y_{j-2}, y_{j-1}\right)},
$$

$$
\vec{c}_{i, 0}\left(\overline{x_{0}, \cdots, x_{i}} ; y_{j}\right)=\frac{x_{i}-x_{i-1}}{\vec{c}_{i-1,0}\left(\overline{x_{0}, \cdots, x_{i-2}, x_{i}} ; y_{j}\right)-\vec{c}_{i-1,0}\left(\overline{x_{0}, \cdots, x_{i-2}, x_{i-1}} ; y_{j}\right)},
$$

$$
\begin{aligned}
\vec{c}_{i, j} & \left(\overline{x_{0}, \cdots, x_{i}} ; y_{0}, \cdots, y_{j}\right) \\
& =\frac{y_{j}-y_{j-1}}{\vec{c}_{i, j-1}\left(\overline{x_{0}, \cdots, x_{i}} ; y_{0}, \cdots, y_{j-2}, y_{j}\right)-\vec{c}_{i, j-1}\left(\overline{x_{0}, \cdots, x_{i}} ; y_{0}, \cdots, y_{j-2}, y_{j-1}\right)} .
\end{aligned}
$$

It is not difficult to prove (see [5]) that $\vec{r}_{n}(x, y) \in \vec{H}_{n^{2}+2 n, 2\left[\left(n^{2}+2 n\right) / 2\right]}$ (here $[x]$ denotes the greatest integer not exceeding $x$ ) and

$$
\vec{r}_{n}\left(x_{i}, y_{j}\right)=\vec{v}_{i, j} \quad(i=0,1, \cdots, n, j=0,1, \cdots, n) .
$$

If the roles of $x$ and $y$ are interchanged, one will obtain a so-called dual Thieletype branched continued fraction (see [5])

$$
\vec{r}_{n}^{*}(x, y)=\vec{t}_{0}^{*}(x)+\frac{y-y_{0}}{\vec{t}_{1}^{*}(x)}+\cdots+\frac{y-y_{n-1}}{\vec{t}_{n}^{*}(x)},
$$


where

$$
\begin{aligned}
\vec{t}_{l}^{*}(x)= & \vec{c}_{0, l}^{*}\left(x_{0} ; \overline{y_{0}, \cdots, y_{l}}\right) \\
& +\frac{x-x_{0}}{\vec{c}_{1, l}^{*}\left(x_{0}, x_{1} ; \overline{y_{0}, \cdots, y_{l}}\right)}+\cdots+\frac{x-x_{n-1}}{\vec{c}_{n, l}^{*}\left(x_{0}, \cdots, x_{n} ; \overline{y_{0}, \cdots, y_{l}}\right)}
\end{aligned}
$$

and $\vec{c}_{i, j}^{*}\left(x_{0}, \cdots, x_{i} ; \overline{y_{0}, \cdots, y_{j}}\right)$ are computed according to the following recursive process

$$
\vec{c}_{0,0}^{*}\left(x_{i}, \bar{y}_{j}\right)=\vec{v}_{i, j} \quad(i=0,1, \cdots, n, j=0,1, \cdots, n),
$$

$$
\begin{aligned}
\vec{c}_{0, j}^{*}( & \left(x_{i} ; \overline{y_{0}, \cdots, y_{j}}\right) \\
& =\frac{y_{j}-y_{j-1}}{\vec{c}_{0, j-1}^{*}\left(x_{i} ; \overline{y_{0}, \cdots, y_{j-2}, y_{j}}\right)-\vec{c}_{0, j-1}^{*}\left(x_{i} ; \overline{y_{0}, \cdots, y_{j-2}, y_{j-1}}\right)},
\end{aligned}
$$

$$
\begin{aligned}
& \vec{c}_{i, 0}^{*}\left(x_{0}, \cdots, x_{i} ; \bar{y}_{j}\right) \\
& \quad=\frac{x_{i}-x_{i-1}}{\vec{c}_{i-1,0}^{*}\left(x_{0}, \cdots, x_{i-2}, x_{i} ; \bar{y}_{j}\right)-\vec{c}_{i-1,0}^{*}\left(x_{0}, \cdots, x_{i-2}, x_{i-1} ; \bar{y}_{j}\right)},
\end{aligned}
$$

$$
\begin{aligned}
& \vec{c}_{i, j}^{*}\left(x_{0}, \cdots, x_{i} ; \overline{y_{0}, \cdots, y_{j}}\right) \\
& \quad=\frac{x_{i}-x_{i-1}}{\vec{c}_{i-1, j}^{*}\left(x_{0}, \cdots, x_{i-2}, x_{i} ; \overline{y_{0}, \cdots, y_{j}}\right)-\vec{c}_{i-1, j}\left(x_{0}, \cdots, x_{i-2}, x_{i-1} ; \overline{y_{0}, \cdots, y_{j}}\right)} .
\end{aligned}
$$

To distinguish $\vec{r}_{n}(x, y)$ from $\vec{r}_{n}^{*}(x, y)$, we might as well call $\vec{r}_{n}(x, y)$ defined in (1.4) -1.9) an $x / y$-type and $\vec{r}_{n}^{*}(x, y)$ defined in 1.11-1.16) a $y / x$-type. It can be proved that $\vec{r}_{n}^{*}(x, y) \in \vec{H}_{n^{2}+2 n, 2\left[\left(n^{2}+2 n\right) / 2\right]}$ and

$$
\vec{r}_{n}^{*}\left(x_{i}, y_{j}\right)=\vec{v}_{i, j} \quad(i=0,1, \ldots, n, j=0,1, \ldots, n) .
$$

Although both $\vec{r}_{n}(x, y)$ and $\vec{r}_{n}^{*}(x, y)$ are of the same rational type and have the same interpolation properties, one can by no means assert that $\vec{r}_{n}(x, y) \equiv \vec{r}_{n}^{*}(x, y)$, as is shown by a numerical example in [5]. However, if the square point-grid $\Pi^{n}$ is symmetric, by which we mean $x_{i}=y_{i}$ for $i=0,1, \ldots, n$, and the vector-grid $\vec{V}^{n}$ is symmetric, by which we mean $\vec{v}_{i, j}=\vec{v}_{j, i}$ for $i, j=0,1, \ldots, n$, then we can conclude $\vec{r}_{n}(x, y) \equiv \vec{r}_{n}^{*}(y, x)($ see [5]).

In what follows, we only restrict ourselves to the discussion of $x / y$-type bivariate vector valued rational interpolants (BVRI), because the results in $x / y$-type BVRI can easily be transplanted into $y / x$-type.

For convenience, let us simply set

$$
\vec{c}_{i, j}^{(i, j)}=\vec{c}_{i, j}\left(\overline{x_{0}, \ldots, x_{i}} ; y_{0}, \ldots, y_{j}\right) \quad(i=0,1, \ldots, n, j=0,1, \ldots, n) .
$$

Then we have the following algorithm to compute $\vec{r}_{n}(x, y)$.

Algorithm 1.1. This algorithm is carried out according to the following three steps.

a) For $i=0,1, \cdots, n$ and $j=0,1, \cdots, n$, let

$$
\vec{c}_{i, j}^{(0,0)}=\vec{v}_{i, j} .
$$


b) For $j=0,1, \cdots, n, p=1,2, \cdots, n$, and $i=p, p+1, \cdots, n$, let

$$
\vec{c}_{i, j}^{(p, 0)}=\frac{x_{i}-x_{p-1}}{\vec{c}_{i, j}^{(p-1,0)}-\vec{c}_{p-1, j}^{(p-1,0)}} .
$$

c) For $i=0,1, \cdots, n, q=1,2, \cdots, n$, and $j=q, q+1, \cdots, n$, let

$$
\vec{c}_{i, j}^{(i, q)}=\frac{y_{j}-y_{q-1}}{\vec{c}_{i, j}^{(i, q-1)}-\bar{c}_{i, q-1}^{(i, q-1)}} .
$$

It is easy to verify that

$$
\vec{r}_{n}\left(x_{i}, y_{j}\right)=\vec{v}_{i, j}, \quad \forall\left(x_{i}, y_{j}\right) \in \Pi^{n} .
$$

Definition 1.3. A vector-grid $\vec{V}^{n}$ is said to be well-defined if the $\vec{c}_{i, j}^{(p, q)}$ as defined in Algorithm 1.1 satisfy $\vec{c}_{i, j}^{(p-1,0)} \neq \vec{c}_{p-1, j}^{(p-1,0)}$ for $j=0,1, \ldots, n, p=1,2, \ldots, n$, and $i=p, p+1, \ldots, n$, and $\vec{c}_{i, j}^{(i, q-1)} \neq \vec{c}_{i, q-1}^{(i, q-1)}$ for $i=0,1, \ldots, n, q=1,2, \ldots, n$, and $j=q, q+1, \ldots, n$. Otherwise the grid $\vec{V}^{n}$ is said to be ill-defined.

It is clear that if a vector-grid $\vec{V}^{n}$ is ill-defined, then Algorithm 1.1 does not work any more.

Example 1.1. Let $\Pi^{1}$ and a two-dimensional vector-grid $\vec{V}^{1}$ be given as follows:

$$
\begin{array}{ccc}
\Pi^{1}: & (1,0) & (1,1) \\
& (0,0) & (0,1), \\
& & \\
\vec{V}^{1}: & (1,0) & (1,1) \\
& (0,0) & (1,0) .
\end{array}
$$

Proceeding by Algorithm 1.1, we obtain

$$
\begin{array}{lll}
\vec{c}_{0,0}^{(0,0)}=(1,0) & & \vec{c}_{0,1}^{(0,0)}=(1,1) \\
\vec{c}_{1,0}^{(0,0)}=(0,0) & & \vec{c}_{1,1}^{(0,0)}=(1,0) \\
& \downarrow & \\
\vec{c}_{0,0}^{(0,0)}=(1,0) & & \vec{c}_{0,1}^{(0,0)}=(1,1) \\
\vec{c}_{1,0}^{(1,0)}=(1,0) & & \vec{c}_{1,1}^{(1,0)}=(0,1) \\
& \downarrow & \\
\vec{c}_{0,0}^{(0,0)}=(1,0) & & \vec{c}_{0,1}^{(0,1)}=(0,1) \\
\vec{c}_{1,0}^{(1,0)}=(1,0) & & \vec{c}_{1,1}^{(1,1)}=\left(-\frac{1}{2}, \frac{1}{2}\right) .
\end{array}
$$

Obviously $\vec{V}^{1}$ is well-defined. As a result,

$$
\begin{aligned}
& \vec{t}_{0}(y)=\vec{c}_{0,0}+\frac{y-y_{0}}{\vec{c}_{0,1}}=(1,0)+\frac{y}{(0,1)}=(1, y), \\
& \vec{t}_{1}(y)=\vec{c}_{1,0}+\frac{y-y_{0}}{\vec{c}_{1,1}}=(1,0)+\frac{y}{\left(-\frac{1}{2}, \frac{1}{2}\right)}=(1-y, y) .
\end{aligned}
$$

Consequently we get

$$
\begin{aligned}
\vec{r}_{1}(x, y) & =\vec{t}_{0}(y)+\frac{x-x_{0}}{\vec{t}_{1}(y)}=(1, y)+\frac{x-1}{(1-y, y)} \\
& =\frac{\left(y^{2}+(1-y)^{2}+(x-1)(1-y), y\left(y^{2}+(1-y)^{2}+x-1\right)\right)}{(1-y)^{2}+y^{2}} .
\end{aligned}
$$


Example 1.2. Let $\Pi^{2}$ and the two-dimensional vector-grid $\vec{V}^{2}$ be given as follows:

$$
\begin{array}{clll}
\Pi^{2}: & (0,0) & (0,1) & (0,2) \\
& (-1,0) & (-1,1) & (-1,2) \\
& (-2,0) & (-2,1) & (-2,2) \\
& & & \\
\vec{V}^{2}: & (2,2) & (6,0) & (24,24) \\
& (12,6) & (6,0) & (12,6) \\
& (0,0) & (6,0) & (-2,2) .
\end{array}
$$

We see $\vec{v}_{0,1}=\vec{v}_{1,1}=\vec{v}_{2,1}=(6,0)$, which leads to $\vec{c}_{0,1}^{(0,0)}=\vec{c}_{1,1}^{(0,0)}=\vec{c}_{2,1}^{(0,0)}$; therefore $\vec{V}^{2}$ is ill-defined and we cannot use Algorithm 1.1 to construct a vector-valued rational function $\vec{r}_{2}(x, y)$ that interpolates $\vec{V}^{2}$ over $\Pi^{2}$.

In the next section, we define a new interpolant with a corresponding algorithm more reliable than Algorithm 1.1.

\section{The Definition And COMputation of BCVRI}

Let us decompose the grid $\Pi^{n}$ into the following two triangular grids:

$$
\begin{array}{cccc}
\left(x_{0}, y_{0}\right) & & & \\
\left(x_{1}, y_{0}\right) & \left(x_{1}, y_{1}\right) & & \\
\vdots & \vdots & \ddots & \\
\left(x_{n}, y_{0}\right) & \left(x_{n}, y_{1}\right) & \cdots & \left(x_{n}, y_{n}\right)
\end{array}
$$

and

$$
\begin{array}{cccc}
\left(x_{0}, y_{1}\right) & \left(x_{0}, y_{2}\right) & \cdots & \left(x_{0}, y_{n}\right) \\
& \left(x_{1}, y_{2}\right) & \cdots & \left(x_{1}, y_{n}\right) \\
& & \ddots & \vdots \\
& & & \left(x_{n-1}, y_{n}\right)
\end{array}
$$

denoted by LB and RU, respectively. We hope to use the policy of "divide and conquer" to construct a kind of composite vector valued rational interpolant. In what follows we abbreviate the term bivariate composite vector valued rational interpolant as BCVRI.

Let

$$
\begin{aligned}
& \vec{R}_{n}(L B ; x, y)=\vec{S}_{0}(L B ; y)+\frac{x-x_{n}}{\mid \vec{S}_{1}(L B ; y)}+\cdots+\frac{x-x_{1}}{\vec{S}_{n}(L B ; y)}, \\
& \vec{R}_{n}(R U ; x, y)=\vec{S}_{0}(R U ; y)+\frac{x-x_{0}}{\vec{S}_{1}(R U ; y)}+\cdots+\frac{x-x_{n-2}}{\vec{S}_{n-1}(R U ; y)}
\end{aligned},
$$

where

$$
\vec{S}_{k}(L B ; y)=\vec{a}_{k, 0}+\frac{y-y_{0}}{\left\lceil\vec{a}_{k, 1}\right.}+\cdots+\frac{y-y_{n-k-1}}{\mid \vec{a}_{k, n-k}}, \quad k=0,1, \cdots, n
$$

$$
\vec{S}_{k}(R U ; y)=\vec{b}_{k, k+1}+\frac{y-y_{k+1}}{\mid \vec{b}_{k, k+2}}+\cdots+\frac{y-y_{n-1}}{\mid \vec{b}_{k, n}}, \quad k=0,1, \cdots, n-1 .
$$


Suppose $\Pi^{n}$ is uniform, i.e.,

$$
x_{i-1}-x_{i}=x_{i}-x_{i+1}=y_{i+1}-y_{i}=y_{i}-y_{i-1}, \quad i=1,2, \cdots, n-1,
$$

and let

$$
\begin{aligned}
& P(x, y)=\prod_{i=0}^{n}\left(x+y-x_{n}-y_{i}\right), \\
& Q(x, y)=\prod_{i=0}^{n-1}\left(x+y-x_{i}-y_{n}\right) .
\end{aligned}
$$

It is clear that $P(x, y)$ and $Q(x, y)$ are polynomials of degree $n+1$ and $n$, respectively, and

$$
\begin{array}{lll}
P\left(x_{i}, y_{j}\right)=0, & Q\left(x_{i}, y_{j}\right) \neq 0 & \text { if }\left(x_{i}, y_{j}\right) \in \mathrm{LB} \\
P\left(x_{i}, y_{j}\right) \neq 0, & Q\left(x_{i}, y_{j}\right)=0 & \text { if }\left(x_{i}, y_{j}\right) \in \mathrm{RU} .
\end{array}
$$

When $\Pi^{n}$ is not uniform, by which we mean that the conditions (2.7) are not satisfied, one can also construct polynomials $P(x, y)$ and $Q(x, y)$ such that (2.10) holds. In general, however, the degrees of the polynomials will be much higher. For example,

$$
\begin{aligned}
& P(x, y)=\prod_{\left(x_{i}, y_{j}\right) \in \mathrm{LB}}\left[\left(x-x_{i}\right)^{2}+\left(y-y_{j}\right)^{2}\right] \\
& Q(x, y)=\prod_{\left(x_{i}, y_{j}\right) \in \mathrm{RU}}\left[\left(x-x_{i}\right)^{2}+\left(y-y_{j}\right)^{2}\right]
\end{aligned}
$$

are the polynomials satisfying (2.10) with degree $(n+1)(n+2)$ and $n(n+1)$, respectively.

Now we define a BCVRI over $\Pi^{n}$ as follows:

$$
\vec{R}_{n}(x, y)=Q(x, y) \vec{R}_{n}(\mathrm{LB} ; x, y)+P(x, y) \vec{R}_{n}(\mathrm{RU} ; x, y) .
$$

The following algorithm aims at computing the coefficients $\vec{a}_{k, l}$ and $\vec{b}_{k, l}$ in branched continued fractions $\vec{R}_{n}(\mathrm{LB} ; x, y)$ and $\vec{R}_{n}(\mathrm{RU} ; x, y)$ simultaneously.

Algorithm 2.1. This algorithm proceeds as follows.

a) For $i=0,1, \cdots, n$ and $j=0,1, \cdots, i$, let

$$
\vec{A}_{i, j}^{(0,0)}=\vec{v}_{i, j} / Q\left(x_{i}, y_{j}\right) .
$$

For $i=0,1, \cdots, n-1$ and $j=i+1, i+2, \cdots, n$, let

$$
\vec{B}_{i, j}^{(0,0)}=\vec{v}_{i, j} / P\left(x_{i}, y_{j}\right) .
$$

b) For $j=0,1, \cdots, n, p=1,2, \cdots, n-j$, and $i=j, j+1, \cdots, n-p$, let

$$
\vec{A}_{i, j}^{(p, 0)}=\frac{x_{i}-x_{n-p+1}}{\vec{A}_{i, j}^{(p-1,0)}-\vec{A}_{n-p+1, j}^{(p-1,0)}} .
$$

c) For $i=0,1, \cdots, n, q=1,2, \cdots, i$, and $j=q, q+1, \cdots, i$, let

$$
\vec{A}_{i, j}^{(n-i, q)}=\frac{y_{j}-y_{q-1}}{\vec{A}_{i, j}^{(n-i, q-1)}-\vec{A}_{i, q-1}^{(n-i, q-1)}} .
$$


d) For $j=1,2, \cdots, n, p=1,2, \cdots, j-1$, and $i=p, p+1, \cdots, j-1$, let

$$
\vec{B}_{i, j}^{(p, 0)}=\frac{x_{i}-x_{p-1}}{\vec{B}_{i, j}^{(p-1,0)}-\vec{B}_{p-1, j}^{(p-1,0)}} .
$$

e) For $i=0,1, \cdots, n-1$ and $j=i+1, i+2, \cdots, n$, let

$$
\vec{B}_{i, j}^{(i, i+1)}=\vec{B}_{i, j}^{(i, 0)} \text {. }
$$

f) For $i=0,1, \cdots, n-2, j=i+2, i+3, \cdots, n$, and $q=i+2, i+3, \cdots, j$, let

$$
\vec{B}_{i, j}^{(i, q)}=\frac{y_{j}-y_{q-1}}{\vec{B}_{i, j}^{(i, q-1)}-\vec{B}_{i, q-1}^{(i, q-1)}} .
$$

Theorem 2.1. Let

$$
\begin{aligned}
& \vec{a}_{k, l}=\vec{A}_{n-k, l}^{(k, l)} \\
& \vec{b}_{k, l}=\vec{B}_{k, l}^{(k, l)} \quad(k=0,1, \cdots, n, l=0,1, \cdots, n-k), \\
& (k=0,1, \cdots, n-1, l=k+1, \cdots, n) .
\end{aligned}
$$

Then

$$
\begin{array}{ll}
\vec{R}_{n}(x, y) \in \vec{H}_{\max \left(\partial Q+n^{2}+n-2, \partial P+n^{2}+n-1\right), n^{2}+n-2} & \text { for even } n, \\
\vec{R}_{n}(x, y) \in \vec{H}_{\max \left(\partial Q+n^{2}+n-1, \partial P+n^{2}+n-2\right), n^{2}+n-2} & \text { for odd } n,
\end{array}
$$

where $\partial Q$ and $\partial P$ denote the total degrees of polynomials $Q(x, y)$ and $P(x, y)$, respectively. (In particular, if $\Pi^{n}$ is uniform, then $\partial Q=n$ and $\partial P=n+1$. In this case, $\vec{R}_{n}(x, y) \in \vec{H}_{n^{2}+2 n, n^{2}+n-2}$ for even $n$ and $\vec{R}_{n}(x, y) \in \vec{H}_{n^{2}+2 n-1, n^{2}+n-2}$ for odd n.) Moreover,

$$
\vec{R}_{n}\left(x_{i}, y_{j}\right)=\vec{v}_{i, j} \quad \forall\left(x_{i}, y_{j}\right) \in \Pi^{n} .
$$

Proof. It is not difficult to show by induction that

$$
\begin{array}{lll}
\vec{R}_{n}(L B ; x, y) \in \vec{H}_{\left(n^{2}+2 n\right) / 2,\left(n^{2}+2 n\right) / 2} & \text { for even } n, \\
\vec{R}_{n}(L B ; x, y) \in \vec{H}_{\left(n^{2}+2 n-1\right) / 2,\left(n^{2}+2 n-3\right) / 2} & \text { for odd } n, \\
\vec{R}_{n}(R U ; x, y) \in \vec{H}_{\left(n^{2}-2\right) / 2,\left(n^{2}-4\right) / 2} & & \text { for even } n, \\
\vec{R}_{n}(R U ; x, y) \in \vec{H}_{\left(n^{2}-1\right) / 2,\left(n^{2}-1\right) / 2} & & \text { for odd } n .
\end{array}
$$

Therefore

$$
\begin{array}{ll}
\vec{R}_{n}(x, y) \in \vec{H}_{\max \left(\partial Q+n^{2}+n-2, \partial P+n^{2}+n-1\right), n^{2}+n-2} & \text { for even } n \\
\vec{R}_{n}(x, y) \in \vec{H}_{\max \left(\partial Q+n^{2}+n-1, \partial P+n^{2}+n-2\right), n^{2}+n-2} & \text { for odd } n .
\end{array}
$$

Since $\Pi^{n}=\mathrm{LB} \cup \mathrm{RU},\left(x_{i}, y_{j}\right) \in \Pi^{n}$ implies $\left(x_{i}, y_{j}\right) \in \mathrm{LB}$ or $\left(x_{i}, y_{j}\right) \in \mathrm{RU}$. If $\left(x_{i}, y_{j}\right) \in \mathrm{LB}$, then from (2.5), 2.19) and 2.15) it follows that

$$
\begin{aligned}
\vec{S}_{k}\left(\mathrm{LB} ; y_{j}\right) & =\vec{A}_{n-k, 0}^{(k, 0)}+\frac{y_{j}-y_{0}}{\mid \vec{A}_{n-k, 1}^{(k, 1)}}+\cdots+\frac{y_{j}-y_{j-1}}{\vec{A}_{n-k, j}^{(k, j)}} \\
& =\vec{A}_{n-k, 0}^{(k, 0)}+\frac{y_{j}-y_{0}}{\vec{A}_{n-k, 1}^{(k, 1)}}+\cdots+\frac{y_{j}-y_{j-2}}{\vec{A}_{n-k, j}^{(k, j-1)}} \\
& =\cdots=\vec{A}_{n-k, j}^{(k, 0)} .
\end{aligned}
$$


By (2.3), (2.14) and (2.12) one has

$$
\begin{aligned}
\vec{R}_{n}\left(\mathrm{LB} ; x_{i}, y_{j}\right) & =\vec{A}_{n, j}^{(0,0)}+\frac{x_{i}-x_{n}}{\mid \vec{A}_{n-1, j}^{(1,0)}}+\cdots+\frac{x_{i}-x_{i+1}}{\mid \vec{A}_{i, j}^{(n-i, 0)}} \\
& =\vec{A}_{n, j}^{(0,0)}+\frac{x_{i}-x_{n}}{\vec{A}_{n-1, j}^{(1,0)}}+\cdots+\frac{x_{i}-x_{i+2}}{\vec{A}_{i, j}^{(n-i-1,0)}} \\
& =\cdots=\vec{A}_{i, j}^{(0,0)}=\vec{v}_{i, j} / Q\left(x_{i}, y_{j}\right) .
\end{aligned}
$$

Therefore, by (2.11) one finally gets

$$
\vec{R}_{n}\left(x_{i}, y_{j}\right)=Q\left(x_{i}, y_{j}\right) \vec{R}_{n}\left(\mathrm{LB} ; x_{i}, y_{j}\right)=\vec{v}_{i, j} .
$$

If $\left(x_{i}, y_{j}\right) \in \mathrm{RU}$, then from (2.6), (2.20) and (2.18) it follows that

$$
\begin{aligned}
\vec{S}_{k}\left(\mathrm{RU} ; y_{j}\right) & =\vec{B}_{k, k+1}^{(k, k+1)}+\frac{y_{j}-y_{k+1}}{\mid \vec{B}_{k, k+2}^{(k, k+2)}}+\cdots+\frac{y_{j}-y_{j-1}}{\vec{B}_{k, j}^{(k, j)}} \\
& =\vec{B}_{k, k+1}^{(k, k+1)}+\frac{y_{j}-y_{k+1}}{\mid \vec{B}_{k, k+2}^{(k, k+2)}}+\cdots+\frac{y_{j}-y_{j-2}}{\vec{B}_{k, j}^{(k, j-1)}} \\
& =\cdots=\vec{B}_{k, j}^{(k, k+1)} .
\end{aligned}
$$

Thus, from (2.4), (2.17), (2.16) and (2.13) we get

$$
\begin{aligned}
\vec{R}_{n}\left(\mathrm{RU} ; x_{i}, y_{j}\right) & =\vec{B}_{0, j}^{(0,1)}+\frac{x_{i}-x_{0}}{\mid \vec{B}_{1, j}^{(1,2)}}+\cdots+\frac{x_{i}-x_{i-1}}{\vec{B}_{i, j}^{(i, i+1)}} \\
& =\vec{B}_{0, j}^{(0,0)}+\frac{x_{i}-x_{0}}{\mid \vec{B}_{1, j}^{(1,0)}}+\cdots+\frac{x_{i}-x_{i-1}}{\vec{B}_{i, j}^{(i, 0)}} \\
& =\vec{B}_{0, j}^{(0,0)}+\frac{x_{i}-x_{0}}{\vec{B}_{1, j}^{(1,0)}}+\cdots+\frac{x_{i}-x_{i-2}}{\vec{B}_{i, j}^{(i-1,0)}} \\
& =\cdots=\vec{B}_{i, j}^{(0,0)}=\vec{v}_{i, j} / P\left(x_{i}, y_{j}\right) .
\end{aligned}
$$

Hence, by (2.11) we have

$$
\vec{R}_{n}\left(x_{i}, y_{j}\right)=P\left(x_{i}, y_{j}\right) \vec{R}_{n}\left(\mathrm{RU} ; x_{i}, y_{j}\right)=\vec{v}_{i, j} .
$$

The proof is completed.

\section{The COMPlexity of AlgorithmS}

Instead of (2.1) and (2.2) one can also carry out other triangular decompositions of the square grid, for instance, the decomposition

$$
\begin{aligned}
& \begin{array}{lllll}
\left(x_{1}, y_{0}\right) & \left(x_{0}, y_{0}\right) & \left(x_{0}, y_{1}\right) & \cdots & \left(x_{0}, y_{n}\right)
\end{array} \\
& \begin{array}{lllll}
\left(x_{2}, y_{0}\right) & \left(x_{2}, y_{1}\right) \quad\left(x_{1}, y_{1}\right) & \cdots & \left(x_{1}, y_{n}\right)
\end{array} \\
& \begin{array}{cccccc}
\vdots & \vdots & \ddots & & \ddots & \vdots \\
\left(x_{n}, y_{0}\right) & \left(x_{n}, y_{1}\right) & \cdots & \left(x_{n}, y_{n-1}\right) & & \left(x_{n}, y_{n}\right)
\end{array}
\end{aligned}
$$


and the decomposition, along another diagonal,

$$
\begin{aligned}
& \left(x_{0}, y_{0}\right) \quad \cdots \quad\left(x_{0}, y_{n-1}\right) \quad\left(x_{0}, y_{n}\right) \\
& \left(x_{1}, y_{0}\right) \cdots\left(x_{1}, y_{n-1}\right) \quad\left(x_{2}, y_{n-1}\right) \quad\left(x_{2}, y_{n}\right) \\
& \begin{array}{c}
\vdots \\
\left(x_{n}, y_{0}\right)
\end{array} \\
& \left(\begin{array}{llll}
\left(x_{n}, y_{1}\right) & \cdots & \left(x_{n}, y_{n-1}\right) \quad\left(x_{n}, y_{n}\right) .
\end{array}\right.
\end{aligned}
$$

It is not difficult to define the corresponding BCVRIs based on the above decompositions which interpolate $\vec{V}^{n}$ over $\Pi^{n}$.

For a vector valued continued fraction, the complexity is obviously related to the computation of the Samelson inverses. From (1.3) we know that carrying out a Samelson inversion for a $d$-dimensional vector demands at least $2 d$ operations of multiplications or divisions. Therefore we take the number of Samelson inverses in an algorithm as the criterion for judging whether the algorithm is complicated or not.

Suppose $N_{1}$ and $N_{2}$ are the total numbers of Samelson inverses to be computed for the vector valued rational interpolants of form (2.11) and (1.4)-(1.5), respectively. Then

$$
\begin{aligned}
N_{1} & =\sum_{j=0}^{n} \frac{(n-j)(n-j+1)}{2}+\sum_{i=0}^{n} \frac{i(i+1)}{2}+\sum_{j=1}^{n} \frac{j(j-1)}{2}+\sum_{i=0}^{n-2} \frac{(n-i+1)(n-i)}{2} \\
& =\frac{n(n+1)(n+2)}{6}+\frac{n(n+1)(n+2)}{6}+\frac{(n-1) n(n+1)}{6}+\frac{(n-1) n(n+1)}{6} \\
& =\frac{n(n+1)(2 n+1)}{3} \\
N_{2} & =n(n+1)^{2},
\end{aligned}
$$

which shows that it is $n(n+1)(n+2) / 3$ times more economical to compute the BCVRI $\vec{R}_{n}(x, y)$ in (2.11) yielded by the decomposition (2.1) and (2.2) of $\Pi^{n}$ than to compute (1.4) and (1.5) directly. Therefore at least $2 n(n+1)(n+2) d / 3$ multiplications are saved through our decomposition method.

\section{Numerical EXAmple}

Let us consider again the grid $\Pi^{2}$ and the corresponding vector-grid $\vec{V}^{2}$ in Example 1.2 i.e.,

$$
\begin{array}{clll} 
& (0,0) & (0,1) & (0,2) \\
\Pi^{2}: & (-1,0) & (-1,1) & (-1,2) \\
& (-2,0) & (-2,1) & (-2,2), \\
& & & \\
\vec{V}^{2}: & (2,2) & (6,0) & (24,24) \\
& (12,6) & (6,0) & (12,6) \\
& (0,0) & (6,0) & (-2,2) .
\end{array}
$$

In this case, $\Pi^{2}$ is uniform and $\vec{V}^{2}$ is ill-defined. We mentioned in Example 1.2 that the computational procedure in Algorithm 1.1 breaks down. In fact, $\vec{r}_{2}(x, y)$ does not exist at all in this case. Otherwise, $\vec{r}_{2}(x, y)$ can be written as

$$
\vec{r}_{2}(x, y)=\vec{t}_{0}(y)+\frac{x-x_{0}}{\vec{t}_{1}(y)}+\frac{x-x_{1}}{\vec{t}_{2}(y)} .
$$


Whatever a reordering of the square point-grid $\Pi^{2}$ and vector-grid $\vec{V}^{2}$ is made, we always have a whole column in $\vec{V}^{2}$, entries of which are all equal to $(6,0)$, i.e., $\vec{v}_{0, j}=\vec{v}_{1, j}=\vec{v}_{2, j}=(6,0)$ with some $j$ in $\{0,1,2\}$. Therefore we have

$$
\vec{r}_{2}\left(x_{0}, y_{j}\right)=\vec{r}_{2}\left(x_{1}, y_{j}\right)=\vec{r}_{2}\left(x_{2}, y_{j}\right),
$$

which leads to

$$
\vec{t}_{0}\left(y_{j}\right)+\frac{x_{1}-x_{0}}{\vec{t}_{1}\left(y_{j}\right)}=\vec{t}_{0}\left(y_{j}\right) .
$$

The above relations imply $\left(x_{1}-x_{0}\right) / \vec{t}_{1}\left(y_{j}\right)=0$ which is impossible because $x_{1} \neq x_{0}$.

Next, we turn to the construction of a BCVRI defined in (2.11). By (2.8) and (2.9),

$$
\begin{aligned}
& P(x, y)=(x+y+2)(x+y+1)(x+y), \\
& Q(x, y)=(x+y-2)(x+y-1) .
\end{aligned}
$$

By (2.12) and (2.13), one gets

$$
\begin{array}{ll}
\vec{A}_{0,0}^{(0,0)}=(1,1), & \\
\vec{A}_{1,0}^{(0,0)}=(2,1), & \vec{A}_{1,1}^{(0,0)}=(3,0), \\
\vec{A}_{2,0}^{(0,0)}=(0,0), & \vec{A}_{2,1}^{(0,0)}=(1,0), \quad \vec{A}_{2,2}^{(0,0)}=(-1,1)
\end{array}
$$

and

$$
\begin{aligned}
& \vec{B}_{0,1}^{(0,0)}=(1,0), \vec{B}_{0,2}^{(0,0)}=(1,1), \\
& \vec{B}_{1,2}^{(0,0)}=(2,1) .
\end{aligned}
$$

According to (2.14) and (2.15), one obtains in order

$$
\begin{array}{lll}
\vec{A}_{0,0}^{(1,0)}=(1,1), & & \\
\vec{A}_{1,0}^{(1,0)}=(2 / 5,1 / 5), & \vec{A}_{1,1}^{(1,0)}=(1 / 2,0), & \\
\vec{A}_{2,0}^{(0,0)}=(0,0), & \vec{A}_{2,1}^{(0,0)}=(1,0), & \vec{A}_{2,2}^{(0,0)}=(-1,1), \\
\vec{A}_{0,0}^{(2,0)}=(3 / 5,4 / 5), & & \\
\vec{A}_{1,0}^{(1,0)}=(2 / 5,1 / 5), & \vec{A}_{1,1}^{(1,0)}=(1 / 2,0), & \\
\vec{A}_{2,0}^{(0,0)}=(0,0), & \vec{A}_{2,1}^{(0,0)}=(1,0), & \vec{A}_{2,2}^{(0,0)}=(-1,1), \\
\vec{A}_{0,0}^{(2,0)}=(3 / 5,4 / 5), & \\
\vec{A}_{1,0}^{(1,0)}=(2 / 5,1 / 5), & \vec{A}_{1,1}^{(1,1)}=(2,-4), & \\
\vec{A}_{2,0}^{(0,0)}=(0,0), & \vec{A}_{2,1}^{(0,1)}=(1,0), & \vec{A}_{2,2}^{(0,1)}=(-1,1), \\
\vec{A}_{0,0}^{(2,0)}=(3 / 5,4 / 5), & & \\
\vec{A}_{1,0}^{(1,0)}=(2 / 5,1 / 5), & \vec{A}_{1,1}^{(1,1)}=(2,-4), & \\
\vec{A}_{2,0}^{(0,0)}=(0,0), & \vec{A}_{2,1}^{(0,1)}=(1,0), & \vec{A}_{2,2}^{(0,2)}=(-2 / 5,1 / 5) .
\end{array}
$$


By Theorem 2.1

$$
\begin{aligned}
\vec{S}_{0}(L B ; y) & =\vec{A}_{2,0}^{(0,0)}+\frac{y-y_{0}}{\mid \vec{A}_{2,1}^{(0,1)}}+\frac{y-y_{1}}{\mid \vec{A}_{2,2}^{(0,2)}} \\
& =(0,0)+\frac{y}{\mid(1,0)}+\frac{y-1}{(-2 / 5,1 / 5)} \\
& =\frac{\left(3 y-2 y^{2}, y^{2}-y\right)}{(3-2 y)^{2}+(y-1)^{2}}, \\
\vec{S}_{1}(L B ; y) & =\vec{A}_{1,0}^{(1,0)}+\frac{y-y_{0}}{\vec{A}_{1,1}^{(1,1)}}=(2 / 5,1 / 5)+\frac{y}{(2,-4)} \\
& =\left(\frac{4+y}{10}, \frac{1-y}{5}\right), \\
\vec{S}_{2}(L B ; y) & =\vec{A}_{0,0}^{(2,0)}=(3 / 5,4 / 5) .
\end{aligned}
$$

This leads to

$$
\begin{aligned}
\vec{R}_{2}(L B ; x, y) & =\vec{S}_{0}(L B ; y)+\frac{x-x_{2}}{\vec{S}_{1}(L B ; y)}+\frac{x-x_{1}}{\vec{S}_{2}(L B ; y)} \\
& =\frac{\left(3 y-2 y^{2}, y^{2}-y\right)}{(3-2 y)^{2}+(y-1)^{2}}+\frac{x+2}{\left\lceil\frac{4+y}{10}, \frac{1-y}{5}\right)}+\frac{x+1}{(3 / 5,4 / 5)} \\
& =\frac{\left(3 y-2 y^{2}, y^{2}-y\right)}{(3-2 y)^{2}+(y-1)^{2}}+\frac{(10 x+20)(6 x+y+10,8 x-2 y+10)}{(6 x+y+10)^{2}+(8 x-2 y+10)^{2}} .
\end{aligned}
$$

According to (2.16)-(2.18), one derives

$$
\begin{array}{ll}
\vec{B}_{0,1}^{(0,0)}=(1,0), & \vec{B}_{0,2}^{(0,0)}=(1,1), \\
& \vec{B}_{1,2}^{(1,0)}=(-1,0), \\
\vec{B}_{0,1}^{(0,1)}=(1,0), & \vec{B}_{0,2}^{(0,1)}=(1,1), \\
& \vec{B}_{1,2}^{(1,2)}=(-1,0), \\
\vec{B}_{0,1}^{(0,1)}=(1,0), & \vec{B}_{0,2}^{(0,2)}=(0,1), \\
& \vec{B}_{1,2}^{(1,2)}=(-1,0) .
\end{array}
$$

By Theorem 2.1

$$
\begin{gathered}
\vec{S}_{0}(R U ; y)=\vec{B}_{0,1}^{(0,1)}+\frac{y-y_{1}}{\mid \vec{B}_{0,2}^{(0,2)}}=(1,0)+\frac{y-1}{\mid 0,1)}=(1, y-1), \\
\vec{S}_{1}(R U ; y)=\vec{B}_{1,2}^{(1,2)}=(-1,0),
\end{gathered}
$$


which results in

$$
\begin{aligned}
\vec{R}_{2}(R U ; x, y) & =\vec{S}_{0}(R U ; y)+\frac{x-x_{0}}{\mid \vec{S}_{1}(R U ; y)} \\
& =(1, y-1)+\frac{x}{\square(-1,0)}=(1-x, y-1) .
\end{aligned}
$$

Hence we finally obtain

$$
\begin{aligned}
& \vec{R}_{2}(x, y)=Q(x, y) \vec{R}_{2}(L B ; x, y)+ P(x, y) \vec{R}_{2}(R U ; x, y) \\
&=(x+y-2)(x+y-1)[ \frac{\left(3 y-2 y^{2}, y^{2}-y\right)}{(3-2 y)^{2}+(y-1)^{2}} \\
&\left.+\frac{(10 x+20)(6 x+y+10,8 x-2 y+10)}{(6 x+y+10)^{2}+(8 x-2 y+10)^{2}}\right] \\
&+(x+y+2)(x+y+1)(x+y)(1-x, y-1) .
\end{aligned}
$$

It is easy to verify that $\vec{R}_{2}(x, y)$ interpolates $\vec{V}^{2}$ over $\Pi^{2}$. In our example, the vector-grid $\vec{V}^{2}$ is ill-defined, and, what is more, as mentioned at the beginning of this section, in this case one fails to find a rational interpolant $\vec{r}_{2}(x, y)$ of the form (1.4). However, $\vec{R}_{2}(x, y)$, as a BCVRI defined in (2.11), still exists. Hence, compared with Algorithm 1.1 our new algorithm for BCVRI is more reliable in the sense that it can overcome the nonexistence of some $\vec{r}_{n}(x, y)$, and more economical in the sense that it involves fewer Samelson inverses.

\section{ACKNOWLEDGMENTS}

The authors would like to thank an anonymous referee for his or her valuable suggestions on the rewriting of Section 1, and for other helpful comments.

\section{REFERENCES}

[1] Cuyt A. and Verdonk B., Multivariate rational interpolation, Computing 34 (1985), 41-61. MR 86g:41003

[2] - A review of branched continued fraction theory for the construction of multivariate rational approximants, Appl. Numer. Math. 4 (1988), 263-271. MR 89k:41018

[3] Graves-Morris P.R., Vector valued rational interpolants I, Numer. Math. 42 (1983), 331-348. MR 85a:65020

[4] Siemaszko W., Thiele-type branched continued fractions for two-variable functions, J. Comput. Appl. Math. 9 (1983), 137-153. MR 85h:65024

[5] Zhu G.Q. and Tan J.Q., The duality of bivariate vector valued rational interpolants over rectangular grids, Math. Num. Sin. 3 (1995), 311-320; English transl., Chinese J. Numer. Math. Appl. 17 (1995), no. 4, 75-84. MR 98i:41015

Institute of Applied Mathematics, Hefei University of Technology, Hefei 230009, P. R. China

E-mail address: jqtan@hfut.edu.cn 